Int. J. Odontostomat.,

5(2):119-125, 2011.

\title{
Asociación entre Maloclusiones y Posición de la Cabeza y Cuello
}

\author{
Association between Malocclusion and Position of the Head and Neck
}

Alejandra Aldana P.; Jéssica Báez R.; Carolina Sandoval C.; Cristian Vergara N."; Doris Cauvi L." \& Alejandro Fernández de la Reguera***t

ALDANA, P. A.; BÁEZ, R. J.; SANDOVAL, C. C.; VERGARA, N. C.; CAUVI, L. D. \& FERNÁNDEZ DE LA REGUERA, A. Asociación entre maloclusiones y posición de la cabeza y cuello. Int. J. Odontostomat., 5(2):119-125, 2011.

RESUMEN: Con la finalidad de contrastar la hipótesis nula "no hay asociación entre maloclusiones y alteraciones posturales de cabeza y cuello" se realizó un estudio descriptivo transversal en pacientes que consultaron por tratamiento de ortodoncia. La muestra consistió en 116 pacientes con maloclusión atendidos en el Programa de Especialización en Ortodoncia y Ortopedia Dento Máxilo Facial 2007-2009 de la Facultad de Odontología de la Universidad de Chile. Se analizaron las fichas de cada uno de ellos y se buscó asociación entre los valores del Análisis Cráneo Cervical de Rocabado, el cefalograma, estudio de modelos y la evaluación funcional de la lengua. Se encontró asociaciones estadísticas débiles entre la rotación anti horaria del cráneo (ángulo cráneo vertebral y distancia intervertebral C0-C1 disminuidas), con clase III esqueletal que además tenían rotación posterior mandibular y que eran dólico faciales. Altos valores del triángulo hioideo, se asociaron débilmente, con Clase II esqueletal, junto con alteraciones de la posición de la lengua en reposo y retrusión dentaria. Se concluye que en pacientes con maloclusión existe una asociación estadísticamente débil con alteraciones de la postura craneocervical.

PALABRAS CLAVE: maloclusión, vértebras cervicales, postura.

\section{INTRODUCCIÓN}

Se ha descrito una estrecha relación entre la columna cervical y el complejo cráneo mandíbula, por lo que se espera que los componentes de ambos sistemas tengan la capacidad potencial de influirse de manera recíproca (Dart, 1946).

La evaluación de la postura de cabeza y cuello ha sido tema de estudio, no sólo debido a la relación propuesta existente entre estas estructuras y la presencia de desórdenes temporomandibulares (Gonzalez \& Manns, 1996; Magee, 2002), dolor de cuello y cefaleas (De Lorenzi, 2000), sino también por la relación biomecánica entre la columna cervical, la cabeza y las estructuras dento faciales (Discacciati de Lértora et al., 2006; Ferreira et al., 2000). Investigadores como Huggare \& Harkness (1993), Hellsing et al. (1987), McGuinnes \& McDonald
(2006), D'Attilio et al. (2005), Hong \& Yi (2001), Motoyoshi et al. (2002), Haller (2000) y Miranda et al. (1999) han asociado la columna cervical y morfología maxilofacial.

Rocabado et al. (1982) y Rocabado (1984) proponen un método de evaluación objetivo a través del cual poder evaluar la biomecánica de la relación cráneo mandibular, mediante el estudio cefalométrico cráneo cervical que lleva su nombre.

Se define postura como la relación entre un segmento o parte del cuerpo, en relación a otro adyacente y entre todos los segmentos del cuerpo humano (Gonzalez \& Manns). Esto es un indicador de eficiencia biomecánica, equilibrio y coordinación neuromuscular (Magee).

\footnotetext{
Cirujano Dentista Especialista en Ortodoncia y Ortopedia Dento Máxilo Facial, Universidad de Chile, Santiago, Chile.

* Prof. Asistente del Depto. de Prótesis y Especialista en Ortodoncia y Ortopedia Dento Máxilo Facial, Universidad de Chile, Santiago, Chile.

*** Prof. Titular de Ortodoncia y Ortopedia Dento Máxilo Facial y Especialista en Ortodoncia y Ortopedia Dento Máxilo Facial, Universidad de Chile, Santiago, Chile.

Kinesiólogo, Instructor de Ortodoncia y Ortopedia Dento Máxilo Facial, Universidad de Chile, Santiago, Chile.
} 
Los músculos posturales mandibulares son parte de la cadena muscular que permite al individuo permanecer de pie con la cabeza erguida. Cuando se producen cambios posturales, las contracciones musculares a nivel del sistema estomatognático cambian la posición mandibular, debido a que la mandíbula busca y adopta nuevas posiciones ante la necesidad de funcionar. Por lo tanto, una actitud postural incorrecta, es considerada factor etiológico de maloclusiones (Discacciati de Lértora et al.; Hellsing et al.; Huggare \& Harkness; D'Attilio et al.; Hong \& Yi; Haller; Miranda et al.; Motoyoshi et al.).

La asociación entre posición de la cabeza y maloclusiones fue descrita por Schwartz en (1926), quien observó en niños con obstrucción de la vía aérea superior, una postura al dormir, con extensión de la cabeza y postuló que podía ser una razón para el desarrollo de una maloclusión Clase II de Angle (Schwartz, 1926).

Solow \& Tallgren (1977) en su estudio sobre morfología dento alveolar y relación postural de cabeza y cuello, mediante análisis cefalométrico de telerradiografías laterales, reportaron que en estudios previos se ha encontrado una marcada correlación positiva entre las relaciones verticales de los maxila-

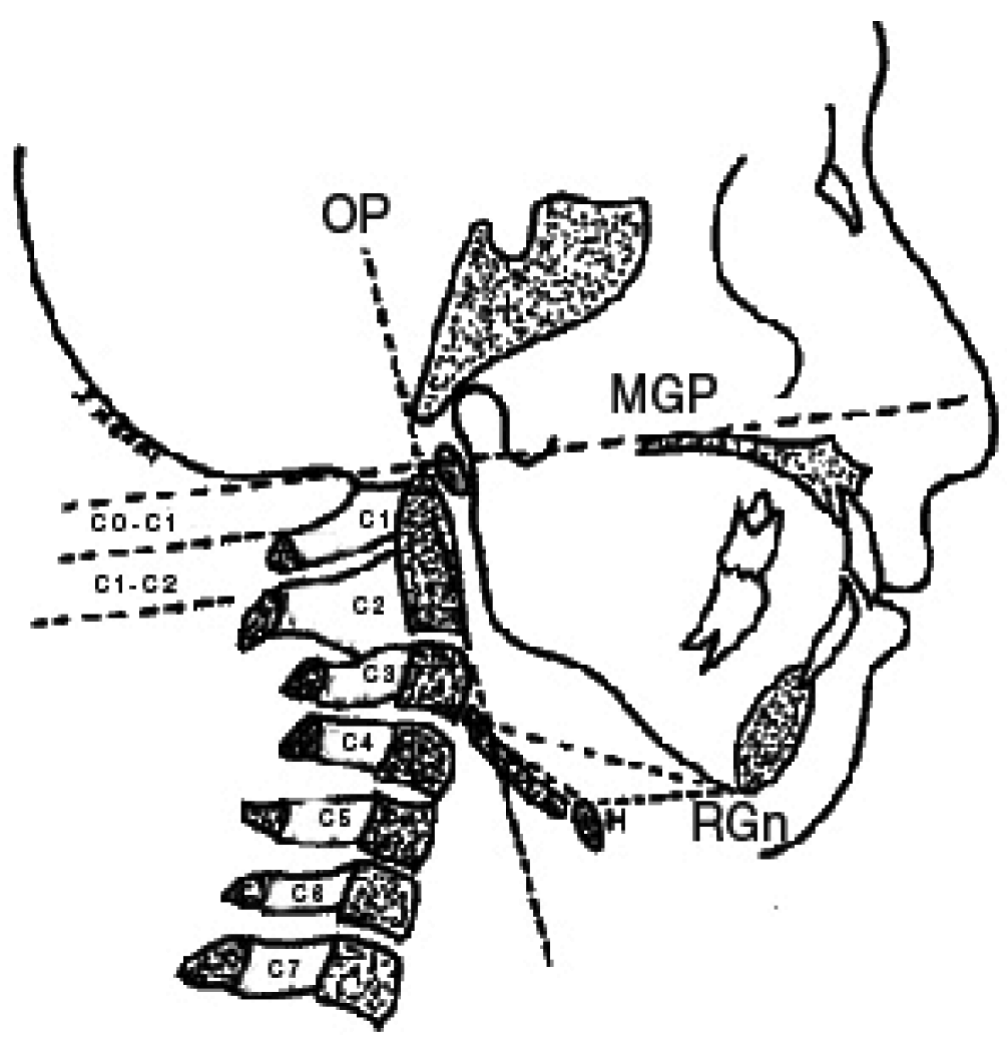

Fig. 1. Análisis cráneo cervical de Mariano Rocabado. res y la posición de la cabeza en relación a la columna cervical, mientras que se ha encontrado una leve correlación entre las relaciones sagitales maxilares y la angulación cráneo cervical.

En 1982 Rocabado et al. establece la asociación entre oclusión de Clase II y postura adelantada de cabeza, descrita según palabras del autor como; "la evidencia más poderosa que ha podido observar en la relación entre maloclusión y posición de la cabeza", lo que motiva a realizar este investigación.

Solow \& Sonnesen (1998) al estudiar la asociación entre postura de cabeza y maloclusiones, en una muestra de noventa y seis niños entre 7-13 años con diagnóstico de maloclusiones severas, usando la clasificación de Björk \& Skieller (1983) mediante análisis cefalométrico, encontraron una clara asociación entre el apiñamiento y la postura cráneo-cervical. Los sujetos con apiñamiento anterior superior e inferior mayor a $2 \mathrm{~mm}$ del arco, tenían ángulos cráneo cervicales mayores en 3-5 grados que los sujetos sin apiñamiento. Estos hallazgos concuerdan con la hipótesis del estiramiento de los tejidos blandos, según la cual el desarrollo sagital de los arcos dentarios es alterado por el aumento de la presión dirigida hacia dorsal en sujetos con postura cráneo cervical extendida. Se postula que una extensión de la postura cráneo cervical lleva a un estiramiento pasivo de los tejidos blandos, comprimiendo la piel, músculos y fascia que cubren cabeza y cuello. Tal mecanismo podría explicar el efecto de la extensión cráneo cervical en el desarrollo del esqueleto facial, en particular, de la mandíbula (Solow \& Sonnesen).

La mayoría de los análisis cefalométricos disponibles evalúan parámetros cráneo máxilo mandibulares, relaciones dentarias entre sí, relaciones dentarias con los maxilares y los tejidos blandos del perfil. Sin embargo no se analiza el componente cervical ni su relación con el cráneo. Al respecto, el análisis descrito por Rocabado, evalúa la estabilidad del cráneo sobre la columna cervical. Según dicho autor, lo fundamental para la interpretación funcional cráneo mandibular es que el paciente durante el proceso de toma de la tele radiografía se encuentre en su posición de reposo habitual para poder pesquisar alteraciones de la biomecánica vertebral (Rocabado) (Fig. 1). 


\section{MATERIAL Y MÉTODO}

Se seleccionó una muestra de 116 pacientes del total de 900 que se encuentran en atención regular en clínica del Postgrado de Ortodoncia y Ortopedia Dento Máxilo Facial de la Facultad de Odontología de la Universidad de Chile, de ambos sexos (35 hombres y 81 mujeres), cuyas edades fluctuaron entre los 7 y 28 años. El método de selección de la muestra fue aleatorio simple sin reposición de los elementos (Tabla I).

Para ser incluídos en el estudio, los pacientes debían tener dentición mixta al menos con la presencia del primer molar, de ambos géneros y de cualquier edad. Debían tener mal oclusión diagnosticada según Clasificación biogenética modificada de la escuela de Bonn. Se excluyeron los pacientes que presentaran tratamiento ortodóncico o quinesiológico previo. También aquellos que presentaran traumatismos cráneo cervicales de importancia y aquellos con condiciones sistémicas que impliquen una alteración postural tales como hipotonía, Síndrome de Down, hipercalcemia, trastornos respiratorios, visuales o de oídos (equilibrio), etc.

Se analizaron las 116 fichas clínicas de los pacientes de la muestra, según los criterios de inclusión y exclusión antes mencionados. Estos pacientes contaban con telerradiografías, modelos de estudio y examen clínico funcional de la posición de la lengua en reposo, fonoarticulación y deglución.

La telerradiografía fue estandarizada en la posición natural de la cabeza mirando al horizonte, descalzo, con la mandíbula en oclusión, sin cefalostato. Todos los registros telerradiográficos fueron tomados con un ortopantomógrafo marca Orthopodos-3. La distancia del tubo de rayos $X$ al film radiográfico fue de $160 \mathrm{~cm}$, la distancia del film al plano medio sagital de la cabeza del paciente fue de $18 \mathrm{~cm}$. El resultado de la magnificación es del $10 \%$. El film es expuesto a 68-70 Kv y $12 \mathrm{~mA}$ con un filtro equivalente de 2 a $5 \mathrm{~mm}$ de aluminio. Se usó una película de $24 \times 30 \mathrm{~cm}$. Todas las telerradiografías fueron tomadas por el mismo radiólogo entrenado, bajo las mismas condiciones para todos los sujetos.

Los exámenes telerradiográficos fueron estudiados con dos protocolos: el análisis cefalométrico utilizado en el Programa de Especialización de Ortodoncia y Ortopedia Dento Máxilo facial de la Universidad de Chile que considera medidas cefalométricas de Steiner (1953), Björk \& Skieller, Shwarz (1961), Jacobson (2003), Burstone (1967), Rikkets (1961), Tweed (1946) y el análisis biomecánico cráneo cervical de Rocabado (Figura 1). Este último análisis considera:

a) El ángulo cráneo vertebral. El componente cráneo cervical vertebral está constituido por la relación funcional del hueso occipital con el atlas (C1) y el axis (C2). En una mecánica normal el occipital se encuentra en una posición de paralelismo con la relación horizontal del atlas. Lo contrario sucede cuando el occipital realiza un movimiento de rotación anterior, donde la base del occipital se aleja del arco posterior del atlas. Este juego que se produce genera un rango de normalidad de $10-11^{\circ}$. Para medir esta relación cráneo vertebral se debe trazar el Plano de McGregor (PMG), Plano odontoideo (PO) y medir el ángulo postero inferior de la intersección $P M G$ y $P O$. Su valor normal es de $96+/-5^{\circ}$.

b) Distancia entre $\mathrm{C} 0-\mathrm{C} 1$, es decir, el espacio de la base del occipital al arco posterior del atlas, que puede variar dentro de rangos funcionales entre 4-9 $\mathrm{mm}$.

c) Distancia entre C1 y C2: esta medida muestra la distancia perpendicular entre el arco posterior del At-

Tabla I. Distribución de la muestra en edad y sexo.

\begin{tabular}{lcccccc}
\hline \multicolumn{1}{c}{ Edad } & Hombres $\mathbf{y}$ mujeres & \multicolumn{2}{c}{ Hombres } & \multicolumn{2}{c}{ Mujeres } \\
& $\mathbf{n}$ & $\%$ & $\mathbf{n}$ & $\%$ & $\mathbf{n}$ & $\%$ \\
\hline $\mathbf{7}$ a $\mathbf{1 2}$ & 56 & 47 & 18 & 51 & 38 & 46 \\
$\mathbf{1 3}$ a $\mathbf{1 8}$ & 47 & 41 & 15 & 43 & 32 & 40 \\
$\mathbf{1 9}$ a $\mathbf{2 4}$ & 10 & 9 & 2 & 6 & 8 & 10 \\
$\mathbf{2 4}$ a $\mathbf{2 9}$ & 3 & 3 & 0 & 0 & 3 & 4 \\
\hline Total & 116 & 100 & 35 & 100 & 81 & 100 \\
\hline
\end{tabular}


las y el proceso espinoso de C2. Las referencias usadas son el punto más posterior e inferior del arco posterior del Atlas y el punto más superior y posterior de la apófisis espinosa de $\mathrm{C} 2$, su valor normal debería ser entre 4-9 $\mathrm{mm}$.

Triángulo hioídeo (medición de su altura). Posición del hueso hioides en relación a la determinación de las curvaturas fisiológicas de la columna cervical. La relación vertical del hueso hioides debe estar por debajo del plano C3-Retrognation (RGn). Esta característica se da cuando existe participación de las estructuras de la región anterior de la columna cervical con una lordosis cervical normal. Cuando se produce una pérdida de las curvaturas fisiológicas cervicales, este triángulo puede hacerse negativo o bien desaparecer. Su valor esperado es de 4+/- 0,6 mm.

Todos los análisis fueron realizados por dos investigadores previamente calibrados para ello. (Indice de Kappa 0,79). Se realizó el trazado cefalométrico en papel de acetato con mina grafito 0,5 y se utilizó una regla milimetrada para mediciones longitudinales y un transportador para mediciones angulares. Los datos obtenidos de la cefalometría ortodóntica nos permitieron determinar la clase esqueletal, el tipo de alteración y su biotipo.

Todos los datos recolectados fueron ingresados a una planilla de cálculo, en la que se tabularon los datos obtenidos. Posteriormente los datos recolectados fueron sometidos a un test de calibración para verificar uniformidad de los investigadores que ejecutaron los análisis. Se aplicó un test de correlación de Pearson para determinar que variables muestran asociación con los parámetros de Rocabado.

Se aplicó un test Shapiro Wilk para determinar que todas las variables tuvieran una distribución normal. Una vez comprobado esto, se aplicó un test de correlación estadística de Pearson demostrándose asociaciones leves, positivas y negativas con valor estadístico respecto de las variables.

\section{RESULTADOS}

El ángulo cráneo vertebral tiene asociaciones estadísticas negativas con el ángulo Silla Articular Gonion (S Ar Go) $(-0,23$ a un $p=0,01)$ y con el Ángulo ANB $(-0,23$ con un $p=0,02)$. Tiene una asociación negativa con el biotipo a través del VERT $(0,26$ con un $p=0,00$ ). Todas estas asociaciones son leves, pero estadísticamente significativas.

El triángulo hioideo tiene una asociación estadística positiva con la lengua en reposo $(0,21$ con un $p=0,02)$. También se encontró una asociación positiva respecto del ANB $(0,23$ con un $p=0,02)$.

La distancia entre C0-C1 tiene asociación negativa con el ángulo Silla Nasion- Gonion Gnation ( $S N-G o G n)(-0,23$ con un $p=0,01)$ y también negativa con el SNB $(-0,21$ con un $p=0,03)$. Finalmente tiene una asociación $(-0,28$ con un $p=0,00)$ con el sexo femenino, es decir que en nuestra muestra los valores más grandes de $\mathrm{C} 0-\mathrm{C} 1$ fueron las mujeres.

La distancia entre C1-C2 tiene una asociación positiva con la edad $(0,21$ con un $p=0,02)$. Tiene una asociación negativa con el ángulo inter incisivo $(-0,22$ con un $p=0,05)$.

\section{DISCUSIÓN}

Dado que nuestros pacientes son atendidos en la universidad es necesario agotar todos los recursos diagnósticos involucrados en la anomalía a tratar. Es por ello que consideramos importante este estudio y asignarle un rol dentro del examen rutinario de cada caso la relación craneovertebral de los pacientes.

El análisis de los datos estableció asociaciones estadísticas entre las alteraciones de la postura cráneo cervical estudiada y las maloclusiones. Estas asociaciones, si bien presentes son leves.

Es importante señalar que al momento de interpretar el análisis de Rocabado 20 , éste debe ser considerado en conjunto, dado que las alteraciones del ángulo cráneo vertebral tienen una estrecha relación con las medidas o distancias entre $\mathrm{C} 0-\mathrm{C} 1$ y C1-C2, entendiéndose que los valores alterados en uno provocarán una reacción en cadena sobre el resto de las estructuras.

En estudios previos se encontró relación estadística entre el ángulo cráneo vertebral y la relación sagital de los molares (Solow \& Tallgren, 1977; Solow \& Sonnesen). En la medida que el ángulo aumenta, los molares tienden a una mesioclusión, esto se puede deber a que al aumentar el ángulo cráneo verte- 
bral flectando la cabeza, se produce una rotación posterior mandibular, la cual para no afectar la vía aérea se compensa con un adelantamiento sagital de la mandíbula con respecto al maxilar. En nuestro estudio no encontramos asociación entre este ángulo y la posición de los molares o caninos.

El ángulo cráneo vertebral mide la posición de la cabeza con respecto a la columna cervical siendo sus valores normales $96^{\circ}+/-5^{\circ}$. Las distancias entre $\mathrm{CO}$ C1 y C1-C2 se aceptan como normales entre 4 y $9 \mathrm{~mm}$ y que sean equivalentes. Esta distancia estará influenciada por la rotación del cráneo con respecto a la columna cervical, así como por la curvatura cervical. Si existe rotación craneal anterior o posterior dichas distancias experimentarán variaciones en sus rangos que serán concordantes con las experimentadas por el ángulo cráneo vertebral y entre ellas mismas.

Otras investigaciones han demostrado una relación entre el ángulo cráneo vertebral, y la distancia entre $\mathrm{C} 0-\mathrm{C} 1$ con el ángulo $\mathrm{SN}-\mathrm{GoG}$ que representa la rotación mandibular. En nuestro estudio la distancia entre $\mathrm{C} 0-\mathrm{C} 1$ se asocia negativamente con una rotación posterior de la mandíbula (ángulo SN-GoGn) y con una posición distal de la mandíbula (con el ángulo SNB). Es decir que en valores pequeños de C0-C1 es esperable encontrar rotación mandibular posterior y clase II.

En el presente estudio el ángulo cráneo vertebral se relacionó negativamente con la rotación anti horaria de la cabeza y los ángulos S-Ar-Go (cef) y $\mathrm{ANB}$, los que disminuían cuando el ángulo cráneo vertebral aumentaba. Esto puede explicar porqué la rotación horaria de la cabeza favorece un crecimiento de tipo vertical.

Lo anterior concuerda con lo reportado en la literatura sobre una asociación entre la postura cráneo cervical y el patrón de crecimiento. Dichos estudios sugieren que la postura de la cabeza sobre la columna cervical puede influir la dirección del crecimiento cráneo facial, posiblemente a través de la teoría del "estiramiento de los tejidos blandos" de Sollow (Sollow \& Tallgren, 1976, 1977; Solow \& Sonnesen).

Según Rocabado en su análisis, existe una asociación estadísticamente significativa entre el patrón facial o biotipo y el ángulo cráneo vertebral. En nuestro estudio encontramos una leve asociación negativa respecto a estas variables. Es decir entre más rotación anti horaria tenía la cabeza y cuello, más dólico era el paciente. Estos hallazgos concuerdan con lo reportado por la literatura, donde Sollow \& Tallgren (1977) determinaron que el patrón dólico facial exhibe un ángulo cráneo cervical aumentado o de valores mayores lo que sería responsable de la postura de la cabeza y el aumento de la resistencia respiratoria (Sollow \& Tallgren, 1977).

Además se asoció la alteración de la posición del hioides con la alteración de la posición de la lengua en reposo y mientras mayor era la distancia del triángulo hioideo había más asociación con clase II esqueletal (ANB).

\section{CONCLUSIONES}

En el presente estudio se encontró asociaciones estadísticas débiles entre los cinco parámetros que analiza el análisis cráneo cervical de Rocabado y los valores encontrados en el examen clínico funcional, examen de modelos y estudio radiográfico de la muestra analizada. Esto corrobora el hecho de que es importante el análisis cráneocervical en los pacientes que presentan anomalías dentomaxilares.

Pudiera considerarse, no como un elemento determinante único, sino dentro de un contexto donde están involucrados otros factores, como un complemento predictivo en los niños de su desarrollo craneofacial futuro. Dado que no pudimos determinar una correlación significativa, no nos es posible asignarle un valor mayor que este.

Existe asociación positiva entre la rotación antihoraria del cráneo (ángulo cráneo-vertebral y distancia inter vertebral $\mathrm{C} 0-\mathrm{C} 1$ disminuidos) y la clase III esqueletal, rotación posterior mandibular y biotipo dólico facial.

Existe asociación estadística positiva entre el triángulo hioideo, Clase II esqueletal y las alteraciones de la posición de la lengua en reposo; y asociación negativa con retrusión incisiva superior e inferior. Consideramos importante decir en este punto, que en ortodoncia la Clase II no es una sola y tiene variaciones (por maxilar, por mandíbula, ambas y funcional).

Existe asociación estadística positiva entre distancia C1-C2 y la variable edad. La asociación fue negativa con ángulo inter incisivo. 
ALDANA, P. A.; BÁEZ, R. J.; SANDOVAL, C. C.; VERGARA, N. C.; CAUVI, L. D. \& FERNÁNDEZ DE LA REGUERA, A. Association between malocclusion and position of the head and neck. Int. J. Odontostomat., 5(2):119-125, 2011.

ABSTRACT: In order to test the null hypothesis "there is no association between malocclusion and postural alterations in head and neck" a non-experimental analytical study was carried out in patients with clinical orthodontic treatment. The sample consisted of 116 patients with malocclusion treated at the Specialization Program in Orthodontics and Dento Maxillo Facial Orthopedics from 2007 to 2009, Faculty of Dentistry, Universidad de Chile. We analyzed the records of each patient, for search partnership between the values of Cervical Skull Rocabado Analysis, the cephalogram and study models. We found weak statistical associations between anti-rotation time of the cranium (skull, vertebral angle and distance diminished intervertebral $\mathrm{C} 0-\mathrm{C} 1$ ), with skeletal class III also had mandibular posterior rotation and were dolicho facial. High values of the hyoid triangle, were weakly associated with skeletal Class II, along with changes in tongue position at rest and dental retrusion. We conclude that in patients with malocclusion there is a statistically weak association with altered craniocervical posture.

KEY WORDS: malocclusion, cervical vertebrae, posture.

\section{REFERENCIAS BIBLIOGRÁFICAS}

Björk, A. \& Skieller, V. Normal and abnormal growth of the mandible. A synthesis of longitudinal cephalometric implant studies over a period of 25 years. Europ. J. Orthodont., 5(1):1-46, 1983.

Burstone, C. J. Lip posture and its significance in treatment planning. Am. J. Orthod., 53(4):262-84, 1967.

D'Attilio, M.; Filippi, M. R.; Femminella, B.; Festa, F. \& Tecco, S. The influence of an experimentally-induced malocclusion on vertebral alignment in rats: a controlled pilot study. Cranio, 23(2):119-29, 2005.

Dart, R. A. The postural aspect of malocclusion. J. Dent. Ass. S. Af., 1(1):1-21, 1946.

De Lorenzi, J. Desarrollo muscular funcional. En: Principios Fundamentales de la Ortopedia Dento-Maxilar. Ohanian, M. C. (Ed.). Caracas, Actualidades Médico-Odontológicas latinoamericanas, 2000. pp.47-9.

Discacciati de Lértora, M. S.; Lértora M. F.; Quintero de Lucas G. V. \& Armella B. Relación entre actitudes posturales y maloclusiones, observadas en adolescentes. Comunicaciones Científicas y Tecnológicas, Universidad Nacional del Nordeste, 2006.

Ferreira, D.; González, M. \& Scheppens, J. Reflexiones sobre la relación entre respiración, postura y disgnacia. Revista A. A. O. F. M., 31(1):7-19, 2000.

Gonzalez, H. E. \& Manns, A. Forward head posture: its structural and functional influence on the stomatognathic system, a conceptual study. Cranio, 14(1):71-80, 1996.
Haller, W. Principios y fundamentos de la ortopedia. En: Principios Fundamentales de la Ortopedia Dento-Maxilar. Ohanian, M. C. (Ed.). Caracas, Actualidades Médico-Odontológicas latinoamericanas, 2000. p.100.

Hellsing, E.; McWilliam, J.; Reigo T. \& Spangfort, E: The relationship between craniofacial morphology, head posture and spinal curvature in 8,11 and 15 year old children. Eur. J. Orthod., 9(4):254-64, 1987.

Hong, S. X. \& Yi, C. K. A classification and characterization of skeletal Class III malocclusion on etio-pathogenic basis. Int. J. Oral Maxillofac. Surg., 30(4):264-71, 2001.

Huggare, J. \& Harkness, E. Associations between head posture and dental occlusion. J. Dent. Res., 72:255, 1993.

Jacobson, A. The "Witts" appraisal of jaw disharmony. 1975. Am. J. Orthod. Dentofacial Orthop., 124(5):470-9, 2003.

Magee, D. Assessment of posture. Orthopedic physical assessment. $4^{\text {th }}$ Ed. St. Louis, Elsevier Sciences, 2002. pp.873-903.

McGuinness, N. J. \& McDonald, J. P. Changes in natural head position observed immediately and one year after rapid maxillary expansion. Eur. J. Orthod., 28(2):126-34, 2006.

Miranda, L.; Trombeta, J. \& Vignola, R. Incidencia de distintas disgnacias. Comprobación Clínica. Revista A. A. O. F. M., 30(1):44-58, 1999. 
Motoyoshi, M.; Shimazaki, T.; Sugai, T. \& Namura, S. Biomechanical influences of head posture on occlusion: an experimental study using finite element analysis. Eur. J. Orthod., 24(4):319-26, 2002.

Ricketts, R. Cephalometrics analysis and synthesis. Angle. Orthod., 31:141-55, 1961.

Rocabado, M.; Johnston, B. \& Blakney, M. Physical therapy and dentistry: an overview. J. Craniomandibular Pract., 1(1):46-9, 1982.

Rocabado, M. Análisis biomecánico cráneo cervical a través de una telerradiografía lateral. Rev. Chil. Ortod., 1:42-52, 1984.

Schwartz, A. M. Kopfhaltung und Kiefer. Zeitschrift für Stomatologie, 24:669-774, 1926.

Schwarz, A. M. Roentgenostatics. A practical evaluation of X-ray headplate. Am. J. Orthod., 47:561-85, 1961.

Sollow, B. \& Tallgren, A. Head posture and craniofacial morphology. Am. J. Phys. Anthropol., 44:417-36, 1976.

Solow, B. \& Tallgren, A. Dentoalveolar morphology in relation to craniocervical posture. Angle Orthod., 47(3):157-64, 1977.

Solow, B. \& Sonnesen, L. Head posture and Malocclusion. Eur. J. Orthod., 20(6):685-93, 1998.

Steiner, C. Cephalometrics for you and me. Am. J. Orthod., 39:729-55, 1953.

Tweed, C. The Frankfort-mandibular plane angle in orthodontic diagnosis, classification, treatment planning, and prognosis. Am. J. Orthod. Oral Surg., 32:175-230, 1946.
Dirección para Correspondencia:

Cristian Vergarana N.

Depto. de Prótesis

Especialista en Ortodoncia y Ortopedia Dento Máxilo Facial Universidad de Chile, Santiago

CHILE

Email: crvergar@u.uchile.cl

Recibido : 06-06-2011

Aceptado: 28-07-2011 